\title{
The Outcomes Of Serial Cerebrospinal Fluid Removal In Elderly Patients With Idiopathic Normal Pressure Hydrocephalus
}

This article was published in the following Dove Press journal: Clinical Interventions in Aging

\author{
Ahmet Turan Isik (D) \\ Derya Kaya (D) \\ Esra Ates Bulut (D) \\ Ozge Dokuzlar' \\ Pinar Soysal (D) ${ }^{2}$ \\ 'Unit for Aging Brain and Dementia, \\ Department of Geriatric Medicine, \\ School of Medicine, Dokuz Eylul \\ University, Izmir, Turkey; ${ }^{2}$ Department of \\ Geriatric Medicine, Bezmialem Vakif \\ University, Faculty of Medicine, Istanbul, \\ Turkey
}

Purpose: Elderly patients with iNPH (idiopathic normal pressure hydrocephalus) might be potentially high-risk surgical patients. Our purpose was to investigate the outcome of serial removal of cerebrospinal fluid (CSF) in the patients with iNPH who refused to have the ventriculoperitoneal or lumboperitoneal shunt surgery or had contraindications to them.

Patients and methods: There were 42 patients, with a median age of 78 years. Recurrent CSF removal was performed when the patients had deteriorated gait which was defined as $>10 \%$ pre-removal change on the average of two walking trials during timed up and go (TUG). All the patients underwent mini-mental status examination (MMSE), frontal assessment battery (FAB), Stroop test, Tinetti Performance Oriented Mobility Assessment (POMA), TUG and nine-hole peg test (NHPT) with the dominant hand, before and after CSF removal.

Results: Thirty-five patients had two CSF removal procedures with a mean interim period of 7.4 months ranging from 1.5 to 35 months. Thirteen patients had three CSF removal procedures. The mean TUG scores were decreased after the first, second and third procedures $(\mathrm{p}<0.001 ; \mathrm{p}<0.001 ; \mathrm{p}=0.007$; respectively). The POMA scores including both gait and balance components improved after the first and second procedures $(p<0.05$; for each). After the third procedure, the increase in POMA-balance score was statistically significant $(p<0.05)$. After the first procedure, the FAB scores and NHPT scores were significantly improved $(\mathrm{p}<0.02)$. The median follow-up duration of the patients was 34.5 months.

Conclusion: The deterioration of gait disturbance may be improved, and cognitive decline may be stabilized, at least postponed, by applying recurrent CSF removal in those unshunted patients with iNPH.

Keywords: normal pressure hydrocephalus, elderly, disability, gait, balance, tap test

\section{Introduction}

Idiopathic normal pressure hydrocephalus (iNPH) is a disease that has a triad of gait disturbance, cognitive impairment and urinary incontinence, and the prevalence increases up to $5.9 \%$ in those older than 80 years. ${ }^{1}$ Albeit the lack of randomized controlled studies, the main treatment of the disease is shunt surgery, and timing of the surgery is so essential that while nearly half of the patients were able to live independently after undergoing shunt surgery at least 6 months from diagnosis, $80 \%$ of those patients who had surgery within 3 months from diagnosis were able to live independently. ${ }^{2}$ Additionally, age is another important factor for the prognosis. It has been reported that approximately $60 \%$ of the patients younger than 75 years
Correspondence: Ahmet Turan Isik Unit for Brain Aging and Dementia, Department of Geriatric Medicine, School of Medicine, Dokuz Eylul University, Balcova, Izmir 35340, Turkey

Tel +902324124341

Fax +902324124349

Email atisik@yahoo.com 
still had gait improvement, whereas $11 \%$ of those older than 75 years demonstrated a favorable gait improvement, by 5 years after the surgery. ${ }^{3}$

As far as comorbidities, frailty, medications and life expectancy of the older adults are concerned, it is clear that some of them might not always be appropriate for the surgery, nor others willing to undergo surgery. In these circumstances, it was reported that gait, balance and cognitive symptoms progress over time in unshunted patients by even 1 year. ${ }^{3}$ In a very recent study, crude 5-year mortality was reported to be $87.5 \%$ in 24 unshunted patients with probable iNPH. Moreover, unshunted individuals with possible iNPH or asymptomatic hydrocephalic ventricular enlargement were found to have a three-fold risk for dementia during 11.5 years of median follow-up. ${ }^{4}$ On the other hand, shunted iNPH patients were also associated with a two to three times higher mortality rate when compared to the general population., ${ }^{3,5}$ It seems that both being shunted and unshunted cause early death and lead to dementia over time in spite of many favorable outcomes of the surgery. Therefore, we hypothesized that the deterioration of gait disturbance and cognition may be stabilized, at least postponed, by applying recurrent cerebrospinal fluid (CSF) removal procedures in those patients who refuse or have contraindication to shunt surgery. To the best of our knowledge, up to now, one retrospective ( $\mathrm{Lim}$ et al) ${ }^{7}$ and one prospective study (Rossi et al) ${ }^{8}$ demonstrated positive experiences of repeated CSF removal procedures. However, the former with 1-year duration of follow-up did not report the time interval between repeated procedures and the latter performed in older patient's ineligible for surgical intervention did not clarify objective functional decline criteria required for deciding CSF removal procedure. Different from these studies, objective criteria were defined as to when to perform CSF removal in the patients with iNPH and the median follow-up duration of the patients was about 3 years in the present study.

This study aimed to investigate the outcome of serial CSF removal procedures in elderly patients with iNPH who refuse or have contraindication to shunt surgery.

\section{Materials And Methods}

\section{Patients}

In this retrospective and follow-up study, patients were recruited from our university hospital between January 2014 and December 2017. Medical records of the patients older than 65 who were diagnosed with probable iNPH according to the international iNPH guidelines (Relkin et al, 2005), ${ }^{9}$ and who refused to have shunt surgery were reviewed and included in the study.

As according to the guideline, ${ }^{9}$ documented cognitive impairment and/or decrease in performance on a cognitive screening instrument, or evidence of at least two of the following deficits (psychomotor slowing; decreased fine motor speed or accuracy; impaired attention; impaired recall; executive dysfunction; behavioral or personality changes) on examination that were not fully attributable to other conditions was required for cognitive impairment.

The exclusion criteria were secondary causes of hydrocephalus, in patients unable to walk, thrombocytopenia $(<50.000)$ or refusal by the patient (technical difficulty for lumbar puncture), incapable of understanding the commands required to do a certain task.

The study was approved by the ethics committee of Dokuz Eylul University School of Medicine (2018/21-27), and participants provided written consent in accordance with the principles of the Declaration of Helsinki.

\section{Measurements}

Forty-two patients underwent comprehensive geriatric assessment including mini-mental state examination test (MMSE), ${ }^{10,11}$ frontal assessment battery (FAB), ${ }^{12}$ Stroop test, ${ }^{13}$ timed up and go (TUG) test, ${ }^{14}$ Tinetti Performance Oriented Mobility Assessment (POMA), ${ }^{15}$ Nine-hole peg test (NHPT) with the dominant hand, ${ }^{16}$ Instrumental Activities of Daily Living (IADL), ${ }^{17}$ Basic Activities of Daily Living $(B A D L)^{21}$ and modified Rankin Scale (mRS), ${ }^{18}$ and brain imaging such as, CT and MRI, were included into the study. The Evan's Index was calculated for each patient.

Cognitive impairment was screened using the MMSE test. $^{10,11}$ The Diagnosis and Statistics Manual of Mental Disorders was used to diagnose dementia. ${ }^{19}$ Urinary incontinence was considered to be present in cases having involuntary urinary leakage in the last 3 months except for urinary tract infection. ${ }^{20}$ The disability of the patients was evaluated by $\mathrm{mRS}$ at the baseline and in the visits following CSF removals. The disability was evaluated by $\mathrm{mRS}$ (range from 0 to 6); 0 = "no symptoms"; 1 = "no significant disability, able to carry out all usual activities"; 2 = "slight disability, able to look after own affairs without assistance"; 3 = "moderate disability, requires some help"; 4 = "moderately severe disability, unable to attend to own bodily needs"; $5=$ "severe disability, requires constant nursing care, bedridden"; 6 = "dead."

All the patients underwent comprehensive geriatric assessment including MMSE, FAB, Stroop test, TUG 
test, POMA, and NHPT with the dominant hand before and after 24 hours of CSF removal.

All patients also underwent cranial imaging before every CSF removal (nearly $30 \mathrm{~mL}$ of CSF was removed). The indication for performing recurrent lumbar puncture was determined in their regular 3-month follow-ups after the diagnosis. When the patients had deteriorated gait which was defined as $>10 \%$ pre-removal change on the average of two walking trials during TUG, CSF removal was conducted.

\section{Statistics}

Statistical analyses were performed with IBM SPSS V.23.0 for Windows (SPSS, IBM Software Group, Chicago, IL, USA). Measurable variables were assessed using the Kolmogorov-Smirnov Goodness-of-Fit test in order to check for normality of distribution for descriptive statistics. Variables with normal distribution were shown as mean $\pm \mathrm{SD}$, and those with non-normal distribution as median (minimum-maximum). Numerical variables were displayed as number of cases, and percent distribution. The Wilcoxon signed ranks test was used for group comparisons. $\mathrm{P}<0.05$ was considered statistically significant.

\section{Results}

There were 42 patients (25 female and 17 male) who underwent recurrent CSF removal procedures, with a median age of 78 years ranging from 62 to 92 . The prevalence of gait and balance impairment, urinary incontinence, and cognitive impairment were $85.7 \%, 78.6 \%$ and $57.1 \%$, respectively. The Hakim's triad was present in $57.1 \%$ of the patients. The median Evan's index was 0.34 ranging from 0.30 to 0.46 . A history of falls in the last 6 months was positive in $69 \%$ of the cases and the median number of falls was one (range 0-6). The median number of nocturia was three. Demographic and clinical features are shown in Table 1.
Table I Demographic And Clinical Features Of The Patients

\begin{tabular}{|l|l|}
\hline Features (n:42) & \\
\hline Age mean+SD (range) & $79.1 \pm 6.9(62-92)$ \\
\hline Gender (F, M) & 25,17 \\
\hline Education year mean+SD (range) & $7.9 \pm 4.6(0-21)$ \\
\hline Charlson's comorbidities index & $1.4 \pm 1.1(0-4)$ \\
\hline $\begin{array}{l}\text { Cerebrovascular risk factors n (\%) } \\
\text { Hypertension } \\
\text { Coronary artery disease } \\
\text { Diabetes mellitus type 2 }\end{array}$ & $34(81)$ \\
\hline Hyperlipidemia & $11(26.2)$ \\
\hline Gait and Balance symptoms $\mathrm{n}(\%)$ & $19(45.2)$ \\
\hline Cognitive symptoms $\mathrm{n}(\%)$ & $12(28.6)$ \\
\hline Urinary incontinence $\mathrm{n}(\%)$ & $36(85.7)$ \\
\hline
\end{tabular}

Thirty-five patients with iNPH had two CSF removal procedures with a mean duration of $7.4 \pm 5.7$ months (range 1.5-35). Thirteen patients had three CSF removal procedures, with a mean duration of $8.5 \pm 3.8$ ranging from 4 to 18 months between second and third procedures. Two patients had a 9-month interval between the third and fourth CSF removal procedure, one with a 6-month interval and one patient with a 3-month interval. The median follow-up duration of the patients was 34.5 (range 5-72) months.

The mean TUG scores were decreased after the first, second and third procedures $(\mathrm{p}<0.001 ; \mathrm{p}<0.001 ; \mathrm{p}<0.02$, respectively). The POMA scores including both gait and balance components improved after the first and second procedures $(\mathrm{p}<0.05$; for each comparison). After the third procedure, the increase in POMA-balance score was statistically significant, but POMA-gait score and POMA total score did not differ before and after the procedure (Table 2).

After the first procedure, the FAB scores and NHPT scores were significantly improved in the patients

Table 2 Outcome Of Gait And Balance Symptoms

\begin{tabular}{|c|c|c|c|c|c|c|c|c|c|}
\hline & \multicolumn{3}{|c|}{ First (n:42) } & \multicolumn{3}{|c|}{ Second (n:35) } & \multicolumn{3}{|c|}{ Third (n: I3) } \\
\hline & Before & After & $\mathbf{P}$ & Before & After & $\mathbf{p}$ & Before & After & $\mathbf{p}$ \\
\hline TUG & $23.3 \pm 15.1$ & $18.8 \pm 9.8$ & $<0.001$ & $29 \pm 29.7$ & $21.8 \pm 20.4$ & $<0.001$ & $21.4 \pm 12.8$ & $19.1 \pm 10.8$ & 0.007 \\
\hline POMA_gait & $8.3 \pm 2.6$ & $9.6 \pm 2.5$ & $<0.001$ & $7 \pm 3.2$ & $8.5 \pm 2.8$ & 0.001 & $9.6 \pm 2.7$ & $10 \pm 1.5$ & 0.498 \\
\hline POMA_balance & $10.1 \pm 4.4$ & II. $5 \pm 4.6$ & $<0.001$ & $9.3 \pm 4.4$ & II. $5 \pm 4.4$ & $<0.001$ & $12 \pm 2$ & $13.8 \pm 1.2$ & 0.042 \\
\hline POMA_total & $18.4 \pm 6.5$ & $21 \pm 6.9$ & $<0.001$ & $16.5 \pm 7.3$ & $20 \pm 6.9$ & 0.001 & $21.6 \pm 3.6$ & $23.8 \pm 2.4$ & 0.066 \\
\hline
\end{tabular}

Note: Data are presented as mean \pm SD.

Abbreviations: TUG, timed up and go test; POMA, Tinetti Performance Oriented Mobility Assessment. 
Table 3 Outcome Of Cognitive Functions

\begin{tabular}{|c|c|c|c|c|c|c|c|c|c|}
\hline & \multicolumn{3}{|c|}{ First (n:42) } & \multicolumn{3}{|c|}{ Second $(n: 35)$} & \multicolumn{3}{|c|}{ Third (n:I3) } \\
\hline & Before & After & $\mathbf{p}$ & Before & After & $\mathbf{p}$ & Before & After & $\mathbf{P}$ \\
\hline MMSE & $21.1 \pm 6.3$ & - & & $21.2 \pm 5.4$ & - & $0.77^{\mathrm{a}}$ & $23.2 \pm 5.7$ & - & $0.676^{b}$ \\
\hline $\mathrm{FAB}$ & $10.0 \pm 4.6$ & $10.1 \pm 4.7$ & 0.012 & $10.5 \pm 4.1$ & $12.0 \pm 3.9$ & 0.553 & $11.5 \pm 4.5$ & $9.6 \pm 5.7$ & \\
\hline NHPT* & $31.0 \pm 9.5$ & $29.7 \pm 6.1$ & 0.008 & $29.7 \pm 6.0$ & $29.7 \pm 6.0$ & 0.374 & $27.3 \pm 7.0$ & $25.7 \pm 6.0$ & \\
\hline CDT & $3.5 \pm 1.5$ & $3.2 \pm 1.5$ & 0.102 & $3.6 \pm 1.5$ & $3.4 \pm 1.5$ & 0.102 & & & \\
\hline ST-dd & $47.6 \pm 13.5$ & $41.5 \pm 8.2$ & 0.600 & $49.3 \pm 18.4$ & $48.6 \pm 15.5$ & 0.398 & $57.2 \pm 23.6$ & $49.9 \pm 22.6$ & \\
\hline ST-e & $4.2 \pm 3.5$ & $4 . I \pm 3.7$ & 1.000 & $4.3 \pm 3.5$ & $4.7 \pm 4.3$ & 0.752 & $3.5 \pm 2.3$ & $1.0 \pm 0.0$ & \\
\hline ST-sc & $2.7 \pm 1.5$ & $1.1 \pm 1.2$ & 0.066 & $2.4 \pm 1.6$ & $1.4 \pm 1.1$ & 0.593 & $3.2 \pm 2.1$ & $1.5 \pm 2.1$ & \\
\hline BADL & $75.3 \pm 22.3$ & & & $72.2 \pm 21.4$ & & $0.526^{c}$ & $65.4 \pm 30.7$ & & $0.789^{d}$ \\
\hline IADL & $13.0 \pm 6.7$ & & & $12.4 \pm 7.4$ & & $0.905^{c}$ & $\mid 3.1 \pm 8.8$ & & $0.858^{d}$ \\
\hline
\end{tabular}

Notes: *Dominant hand; ${ }^{a} \mathrm{p}$ for the comparison of before the first and before the second CSF removal procedures ( $\mathrm{n}=32$ ); ${ }^{\mathrm{p}} \mathrm{p}$ for the comparison of before the first and before the third procedures $(n=9)$; ${ }^{c} p$ for the comparison of before the first and the second procedures $(n=35)$; ${ }^{d} p$ for the comparison of before the second and the third procedures $(n=13)$. Data are presented as mean \pm SD.

Abbreviations: BADL, Basic Activities of Daily Living; CDT, Clock drawing test; FAB, Frontal assessment battery; dd, difference of duration in seconds; e, numbers of errors; IADL, Instrumental Activities of Daily Living; MMSE, Mini-mental state examination test; NHPT, Nine-hole peg test; sc, numbers of spontaneous correction; ST, Stroop Test.

$(\mathrm{p}=0.012)$. However, the scores of the two tests did not differ following the second and the third CSF removal procedures. We observed no changes in the MMSE, STROOP test scores and ADLs during the whole study. (Table 3).

There were only two patients, one of them had complicated Intracranial Hypotension-Like Syndrome, ${ }^{20}$ having headache after lumbar puncture that had resolved with hydration and analgesics. At the last follow-up, incontinence had disappeared in eight patients (19.0\%), and was reduced in seven patients $(16.6 \%)$, continued in the same way in 11 patients $(26.1 \%)$ and 16 patients $(38.0 \%)$ started to wear diapers. The distribution of the degree of disability of the patients at the baseline and following the first (mRS1) and second (mRS2) CSF removal evaluated by scores is shown in Figure 1.

\section{Modified Rankin Scale Score Distrubition}

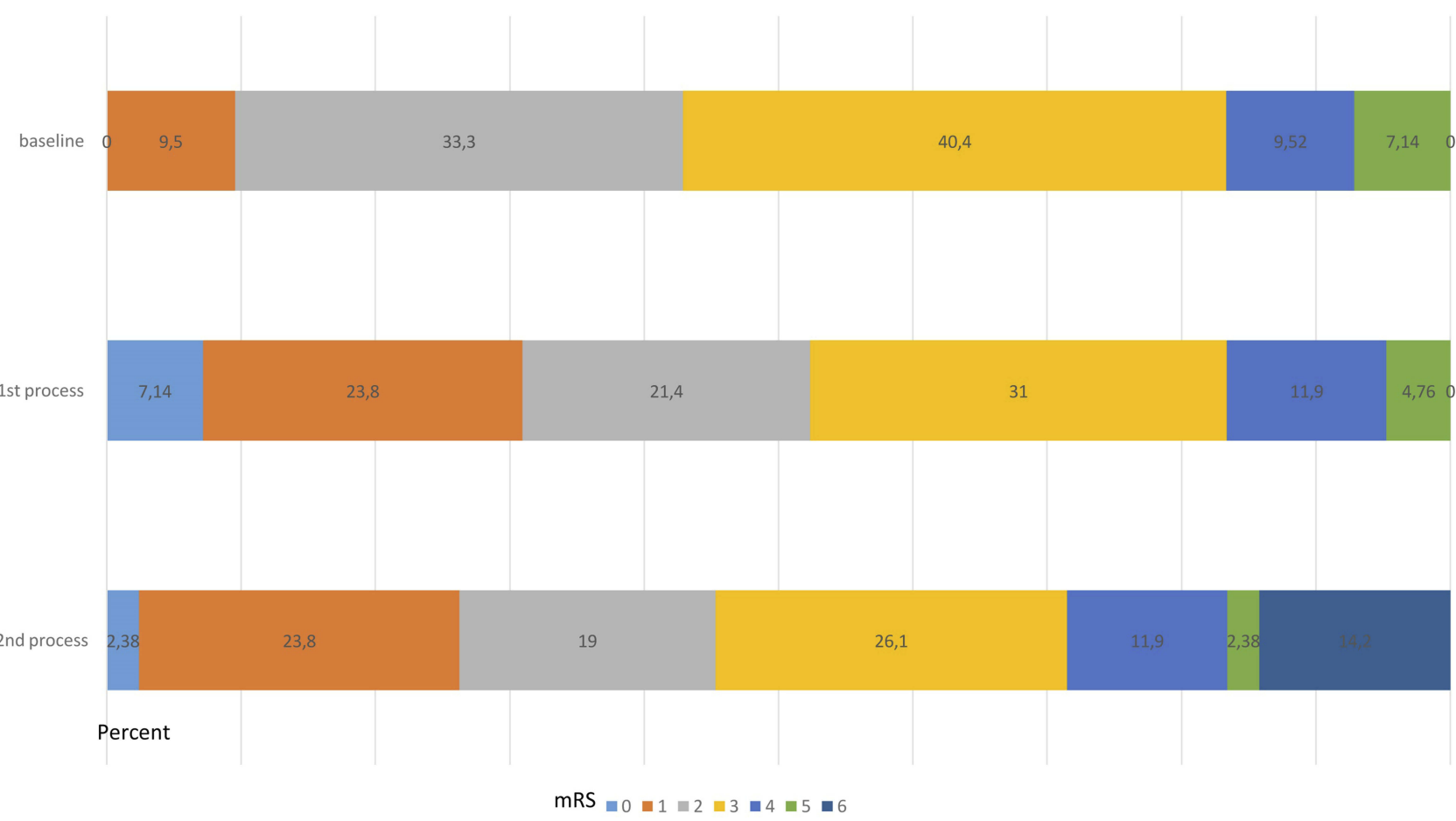

Figure I Modified Rankin Scale score distribution of the patients with iNPH. 


\section{Discussion}

In this retrospective observational study, it was demonstrated that serial CSF removal in older adults with NPH not only improved gait-balance impairment and degree of disability, but cognitive functions and ADLs had also not deteriorated during a follow-up of 34.5 months.

Ventricular enlargement was found to be associated with increased intraventricular pulse pressure despite normal mean CSF pressure in $\mathrm{NPH}^{22}$ Including but not limited to the mechanism, our approach might have led to a reduction in tangential shearing forces on the paracentral fibers of the corona radiate in view of the fact that the forces are considered to produce the well-known clinical trial. ${ }^{23,24}$ In previous studies, it has been shown that iNPH is a progressive disease when left untreated, ${ }^{2,25}$ and shunt surgery is still the main treatment of the disease ${ }^{2}$ even though it cannot completely prevent the patients from progressing to dementia. ${ }^{5,6}$ In a recent prospective longitudinal observational study, symptomatic benefits offered by ventriculoperitoneal shunt (VPS) were partial and transient and treatment might be associated with a high complication rate and a poor long-term functional dependence, especially in older patients. ${ }^{26}$ It was reported that approximately $25 \%$ of the patients had major or minor complications such as, intracranial hematoma, subdural effusion, subdural hematoma, infection, shunt malfunction, over drainage, and hypotensive headaches, after the shunt surgery. ${ }^{27}$ In addition, it was reported that lumboperitoneal shunting, an effective alternative surgery to cranial surgery, improved the mRS score in most of the patients. But even with that technique there were serious adverse events in a fifth of the patients 1 year after the surgery. ${ }^{28}$

In addition, in spite of initial improvement, delayed symptom progression including gait, cognitive, and urinary symptoms, was reported in half of the patients after shunt surgery, which is more likely for elderly patients. ${ }^{29}$ There is no pharmacologically approved agent used in the iNPH treatment today ${ }^{30}$ if anecdotally given acetazolamide is not taken into account, which has limited effectivity ${ }^{29}$ and side effects such as metabolic acidosis, kidney stone, hypokalemia, tingling and fatigue in older adults. ${ }^{31}$

Moreover, it should be kept in mind that older adults might be potentially high-risk surgical patients, ${ }^{30}$ and some of the patients may refuse the surgery although they were informed about the risk of dementia and shortening of lifetime, as reported in the literature..$^{25,26,32}$
Therefore, the development of alternative treatment approaches such as serial CSF removal is crucial for the elderly patients who refuse or have contraindication to shunt surgery. In the present study, it was demonstrated that serial CSF removal improves the walking speed, as in previous studies, ${ }^{19,33-35}$ and gait and balance, but also has positive effects on psychomotor rate and cognitive test. Additionally, it improves the bladder control in $35 \%$ of the patients, as well. It is quite important that all these improvements were accomplished without any deterioration either in the cognitive functions or in the ADLs of the patients. In our opinion, this procedure can transiently decrease in periventricular tensile strength caused by chronic periventricular ischemia, one of those potential mechanisms in the pathophysiology of iNPH and prevent the brain from increasing compliance of the ventricular wall and gradual ventricular enlargement. ${ }^{36}$ For this reason, with this approach, it may be possible that the progression to dementia might be postponed or slowed down, and thus helping older adults with NPH suffering from gait and balance problems, urinary incontinence and memory complaints. In addition, these beneficial effects of serial CSF removal were also reflected in the disability degrees of the cases, which showed a change in the direction of improvement. This is a highly satisfying outcome for the patients with NPH, considering that the proportion with a favorable outcome (defined as a mRS score of 0 or 1 at 90 days) for patients with acute ischemic stroke assigned to treatment with intravenous tissue plasminogen activator within 3 hours of symptom onset, was reported as $33 \%{ }^{37}$

Given the fact that delayed symptom progression occurs about 2 years after shunt surgery, ${ }^{29}$ which is the only treatment of the disease, according to our results after approximately 3 years of follow-up, it is better understood how serial CSF removal can be important for geriatric practice.

Lim et $\mathrm{al}^{7}$ pointed out that repetitive lumbar punctures could maintain symptomatic relief up to 3 months after 1 year of observation in some patients with NPH. Meanwhile, Rossi et al, ${ }^{8}$ in a well-designed prospective study, showed that older patients ineligible for surgical intervention in a geriatric unit benefitted from repeated CSF taps with a time frame of 7 months between CSF removal procedures, with very low minor side effects and without major ones, both of which were consistent with our study. Also, as in our study, they reported that most responsive symptoms to the repeated taps were walking speed and gait disturbances. Moreover, our results were in accordance with the finding that the treated 
group tended to remain stable under a functional and cognitive viewpoint and the cognitive functions were evaluated by the Stroop test and FAB as well as MMSE in the present study. However, our results seem more realistic than those of Rossi et al because they excluded the patients who did not benefit from the first CSF tap procedure, called the nonresponder group, and considered this lack of response as challenging the diagnostic hypothesis of iNPH. In this study, all the patients diagnosed with iNPH continued to undergo CSF removals, without questioning the possible benefits of the first procedure, in order that true iNPH cases might be non-responder for the CSF removals but benefit from the surgery. ${ }^{40}$ Our patients kept undergoing the CSF tap procedures as far as possible because they didn't have a second-choice. Additionally, in that study subsequent CSF removals were scheduled on an individual basis according to the observed functional decline and this decline was not clearly defined in contrast to our study.

The powerful aspect of the present study is that it is one of the first studies to evaluate serial CSF removal in older adults with iNPH by setting objective criteria for stating when to perform repeated CSF taps and detailed cognitive outcomes after the procedures during the followup. Nevertheless, the present study has some limitations. First, the sample size was too small to generalize the results. Second, the lack of shunted patients. Lastly, as the lumbar puncture was done when the patients already had deteriorated gait which was defined as $>10 \%$ preremoval change on the walking trials during TUG, it was not easy to count the follow up time for patients who received three punctures from the moment of the first puncture. Despite these limitations, we think that our findings deserve attention when patients with iNPH refuse and/or have contraindication to shunt surgery.

\section{Conclusion}

The deterioration of gait disturbance, the hallmark of the disease, may be improved and cognitive decline may be stabilized, at least delayed, by applying recurrent CSF removal in those unshunted patients with iNPH. From another point of view one can think that the approach was better than doing nothing in such a progressive disease. The complication rate of the process was very low with no infection. The current study needs to be confirmed with a higher number of patients and with a longer period of follow-up. Also, further studies should be performed for comparing the outcome of the surgery and the serial lumbar punctures.

\section{Disclosure}

The authors report no conflicts of interest in this work. The study received no funding support.

\section{References}

1. Jaraj D, Rabiei K, Marlow T, Jensen C, Skoog I, Wikkelsø C. Prevalence of idiopathic normal-pressure hydrocephalus. Neurology. 2014;82 (16):1449-1454. doi:10.1212/WNL.0000000000000342

2. Andren K, Wikkelso C, Tisell M, Hellstrom P. Natural course of idiopathic normal pressure hydrocephalus. J Neurol Neurosurg Psychiatry. 2014;85:806-810. doi:10.1136/jnnp-2013-306117

3. Kahlon B, Sjunnesson J, Rehncrona S. Long-term outcome in patients with suspected normal pressure hydrocephalus. Neurosurgery. 2007; 60:327-332. doi:10.1227/01.NEU.0000249273.41569.6E

4. Jaraj D, Wikkelsø C, Rabiei K, et al. Mortality and risk of dementia in normal-pressure hydrocephalus: a population study. Alzheimers Dement. 2017;13:850-857. doi:10.1016/j.jalz.2017.01.013

5. Tisell M, Hellström P, Ahl-Börjesson G, et al. Long-term outcome in 109 adult patients operated on for hydrocephalus. Br J Neurosurg. 2006;20:214-221. doi:10.1080/02688690600852324

6. Malm J, Kristensen B, Stegmayr B, Fagerlund M, Koskinen LO. Three-year survival and functional outcome of patients with adult hydrocephalus syndrome. Neurology. 2000;55:576-578. doi:10.1212/ wn1.55.4.576

7. Lim TS, Yong SW, Moon SY. Repetitive lumbar punctures as treatment for normal pressure hydrocephalus. Eur J Neurol. 2009;62:293297. doi: $10.1159 / 000235808$

8. Rossi PD, Damanti S, Nani C, et al. Repeated cerebrospinal fluid removal procedure in older patients with idiopathic normal pressure hydrocephalus ineligible for surgical treatment. J Am Med Dir Assoc. 2019;20:373-376. doi:10.1016/j.jamda.2018.11.014

9. Relkin N, Marmarou A, Klinge P, Bergsneider M, Black PM. Diagnosing idiopathic normal-pressure hydrocephalus. Neurosurgery. 2005;57(3 Suppl):S4-S16; discussion ii-v. doi:10.1227/01.neu.00 00168185.29659.c5

10. Folstein MF, Folstein SE, McHugh PR. "Mini-mental state". A practical method for grading the cognitive state of patients for the clinician. J Psychiatr Res. 1975;12:189-198. doi:10.1016/0022-3956(75) 90026-6

11. Gungen C, Ertan T, Eker E, Yasar R, Engin F. Reliability and validity of the standardized Mini Mental State Examination in the diagnosis of mild dementia in Turkish population. Turk Psikiyatri Derg. 2002;13:273-281

12. Dubois B, Slachevsky A, Litvan I, Pillon B. The FAB: a frontal assessment battery at bedside. Neurology. 2000;55:1621-1626. doi:10.1212/ wnl.55.11.1621

13. Stroop JR. Studies of interference in serial verbal reactions. $J$ Exp Psychol. 1935;18:643-662. doi:10.1037/h0054651

14. Podsiadlo D, Richardson S. The timed 'Up \& Go': a test of basic functional mobility for frail elderly persons. $J$ Am Geriatr Soc. 1991;39:142-148. doi:10.1111/j.1532-5415.1991.tb01616.x

15. Tinetti ME. Performance-oriented assessment of mobility problems in elderly patients. J Am Geriatr Soc. 1986;34:119-126. doi:10.1111/ j.1532-5415.1986.tb05480.x

16. Oxford Grice K, Vogel KA, Le V, Mitchell A, Muniz S, Vollmer MA. Adult norms for a commercially available nine hole peg test for finger dexterity. Am J Occup Ther. 2003;57:570-573. doi:10.5014/ ajot.57.5.570

17. Lawton M, Brody E. Assessment of older people: self-maintaining and instrumental activities of daily living. Gerontologist. 1969;9: 179-186.

18. Rankin J. Cerebral vascular accidents in patients over the age of 60 . Scott Med J. 1957;2:200-215. doi:10.1177/003693305700200504 
19. American Psychiatric Association. Diagnostic and Statistical Manual of Mental Disorders. 5th. American Psychiatric Association; 2013 Available from: https://dsm.psychiatryonline.org/doi/book/10.1176/ appi.books.9780890425596. Accessed April 24, 2019.

20. Ates Bulut E, Soysal P, Isik AT. Frequency and coincidence of geriatric syndromes according to age groups: single-center experience in Turkey between 2013 and 2017. Clin Interv Aging. 2018;13:1899-1905. doi:10.2147/CIA.S180281

21. Mahoney FI, Barthel DW. Functional evaluation: the Barthel index. Md State Med J. 1965;14:61-65.

22. Ravdin LD, Katzen HL, Jackson AE, Tsakanikas D, Assuras S, Relkin NR. Features of gait most responsive to tap test in normal pressure hydrocephalus. Clin Neurol Neurosurg. 2008;110:455-461. doi:10.1016/j.clineuro.2008.02.003

23. Kaya D, Soysal P, Isik AT. Intracranial hypotension-like syndrome after a spinal tap test performed for idiopathic normal pressure hydrocephalus. Am J Alzheimers Dis Other Demen. 2015;30:569572. doi:10.1177/1533317515576390

24. Graff-Radford NR, Knopman DS, Penman AD, Coker LH, Mosley TH. Do systolic BP and pulse pressure relate to ventricular enlargement? Eur J Neurol. 2013;20:720-724. doi:10.1111/ene.12067

25. Hakim S, Venegas JG, Burton JD. The physics of the cranial cavity, hydrocephalus and normal pressure hydrocephalus: mechanical interpretation and mathematical model. Surg Neurol. 1976;5:187-210.

26. Fisher CM. Hydrocephalus as a cause of disturbances of gait in the elderly. Neurology. 1982;32:1358-1363. doi:10.1212/wnl.32.12.1358

27. Razay G, Vreugdenhil A, Liddell J. A prospective study of ventriculoperitoneal shunting for idiopathic normal pressure hydrocephalus. J Clin Neurosci. 2009;16:1180-1183. doi:10.1016/j.jocn.2009.02.013

28. Illán-Gala I, Pérez-Lucas J, Martín-Montes A, Mánnez-Miró J, Arpa J, Ruiz-Ares G. Long-term outcomes of adult chronic idiopathic hydrocephalus treated with a ventriculo-peritoneal shunt. (Evolución a largo plazo de la hidrocefaliacrónica del adultoidiopáticatratada con válvula de derivaciónventrículo-peritoneal). Neurología. 2017;32:205-212. doi:10.1016/j.nrl.2015.10.002

29. Wilson RK, Williams MA. The role of the neurologist in the longitudinal management of normal pressure hydrocephalus. Neurologist. 2010;16:238-248. doi:10.1097/NRL.0b013e3181de4907

30. Kazui H, Miyajima M, Mori E, Ishikawa M. SINPHONI-2 investigators. Lumboperitoneal shunt surgery for idiopathic normal pressure hydrocephalus (SINPHONI-2): an open-label randomised trial. Lancet Neurol. 2015;14(6):585-594. doi:10.1016/S1474-4422(15)00046-0
31. Benveniste RJ, Sur S. Delayed symptom progression after ventriculoperitoneal shunt placement for normal pressure hydrocephalus. $J$ Neurol Sci. 2018;393:105-109. doi:10.1016/j.jns.2018.08.002

32. Alperin N, Oliu CJ, Bagci AM, et al. Low-dose acetazolamide reverses periventricular white matter hyperintensities in iNPH. Neurology. 2014;82:1347-1351. doi:10.1212/WNL.0000000000000313

33. Chapron DJ, Gomolin IH, Sweeney KR. Acetazolamide blood concentrations are excessive in the elderly: propensity for acidosis and relationship to renal function. J Clin Pharmacol. 1989;29:348-353. doi:10.1002/j.1552-4604.1989.tb03340.x

34. Sankar A, Beattie WS, Wijeysundera DN. How can we identify the high-risk patient? Curr Opin Crit Care. 2015;21:328-335. doi:10. 1097/MCC.0000000000000216

35. Stolze H, Kuhtz-Buschbeck JP, Drücke H, et al. Gait analysis in idiopathic normal pressure hydrocephalus-which parameters respond to the CSF tap test? Clin Neurophysiol. 2000;111:1678-1686. doi:10.1016/s1388-2457(00)00362-x

36. Bugalho P, Guimarães J. Gait disturbance in normal pressure hydrocephalus: a clinical study. Parkinsonism Relat Disord. 2007;13:434437. doi:10.1016/j.parkreldis.2006.08.007

37. Souza RKM, Rocha SFBD, Martins RT, Kowacs PA, Ramina R. Gait in normal pressure hydrocephalus: characteristics and effects of the CSF tap test. Arq Neuropsiquiatr. 2018;76:324-331. doi:10.1590/ 0004-282X20180037

38. Bradley WG Jr, Whittemore AR, Watanabe AS, Davis SJ, Teresi LM, Homyak M. Association of deep white matter infarction with chronic communicating hydrocephalus: implications regarding the possible origin of normal-pressure hydrocephalus. AJNR Am J Neuroradiol. 1991;12:31-39.

39. Emberson J, Lees KR, Lyden P, et al. Effect of treatment delay, age, and stroke severity on the effects of intravenous thrombolysis with alteplase for acute ischaemic stroke: a meta-analysis of individual patient data from randomised trials. Lancet. 2014;384:1929-1935. doi:10.1016/S0140-6736(14)60584-5

40. Marmarou A, Bergsneider M, Klinge P, Relkin N, Black PM. The value of supplemental prognostic tests for the preoperative assessment of idiopathic normal-pressure hydrocephalus. Neurosurgery. 2005;57(3 Suppl):S17-S28; discussion ii-v. doi:10.1227/01.neu.0000168184.01 002.60
Clinical Interventions in Aging

\section{Publish your work in this journal}

Clinical Interventions in Aging is an international, peer-reviewed journal focusing on evidence-based reports on the value or lack thereof of treatments intended to prevent or delay the onset of maladaptive correlates of aging in human beings. This journal is indexed on PubMed Central, MedLine, CAS, Scopus and the Elsevier
Bibliographic databases. The manuscript management system is completely online and includes a very quick and fair peer-review system, which is all easy to use. Visit http://www.dovepress.com/ testimonials.php to read real quotes from published authors. 